\title{
Trans-atrial, Trans-pulmonary Repair of Tetralogy of Fallot in Older Children and Adults:
}

\author{
Koirala B, Koirala R, Sharma J, Pradhan S, Acharya A, Sharma P, Rai K,
}

\section{Introduction:}

Tetralogy of Fallot is the most common cyanotic congenital heart disease and has a favorable outcome if dealt with in time. Here we present the retrospective study of immediate outcome of surgical repair of Fallot's Tetralogy carried out at Shahid Gangalal National Heart Centre, Kathmandu, Nepal.

The data was collected from the patients' hospital records, The parameters that we compiled were the patient demography, echocardiographic study result, angiographic data for presence of MAPCA's, the surgical data and post-operative outcomes. The surgical data comprised the duration of CPB, Aortic cross clamp, type of surgical intervention to relieve RVOT obstruction and post repair pressure studies.

The study period extended from Aug 2001 to Jan 2004. Fifty patients with Tetralogy of Fallot's anomaly were operated in that period. There were 26 patients aged 10 years and above. The male to female ratio was 1.1:1. The mean age was 14.7_+ 3.8 years. Most of the patients were in NYHA II (57.7\%) and 76.9\% had clubbing. The Echo study revealed that the average VSD size was $14.4 \ldots+3.8 \mathrm{~mm}$, aortic override was more than $50 \%$ in $5(19.2 \%)$, PDA was present in $2(7.7 \%)$, ASD in $2(7.7 \%)$, average MPA size was $12.8+6.02 \mathrm{~mm}$. All patients underwent trans-atrial, trans-pulmonary artery correction of the anomaly, with Te flon (Gore-Tex) patch closure of the VSD, and pericardial patch augmentation of the pulmonary artery except in $1(3.8 \%)$ patient, Infundibular augmentation was carried out in $8(30.7 \%)$. Pulmonary neo-cusp creation using pericardium in $1(3.8 \%)$. Intra-operative revision of the correction was carried out in $3(12 \%)$. RVOT patch was added in $2(7.7 \%)$ and RPA augmentation in $1(3.8 \%)$. There were $\sim 2(7.7 \%)$ immediate post-operative deaths. There was one late death due to acute infective endocarditis following VSD patch leak. Thus, total correction of Tetralogy of Fallot is feasible even amongst older children with an acceptable result.

Sahid Gangalal National Heart Centre, Kathmandu, Nepal. 